\title{
Approximation of Quasisymmetries Using Circle Packings
}

\author{
G. Brock Williams* \\ Department of Mathematics, University of Tennessee, \\ Knoxville, Tennessee, USA
}

\begin{abstract}
The Universal Teichmüller Space has recently gained the attention of physicists as a setting for developing string theory. Two common models for this space are the set of quasicircles and the set of quasisymmetries. The link between these models lies in the fact that the Riemann maps from $\mathbb{D}$ and $\mathbb{D}^{*}$ to the complementary domains of quasicircles induce quasisymmetric automorphisms of $\partial \mathbb{D}$. We develop a means of approximating these quasisymmetries given their associated quasicircles. This provides a concrete method for switching from the quasicircle to the quasisymmetry model of the Universal Teichmüller Space. Our approach uses discrete analytic functions induced by circle packings to approximate the Riemann maps.
\end{abstract}

\section{Introduction}

A recurrent theme of complex analysis has been the linking of geometric properties of a curve or surface with the function theoretic properties of an associated map. One of the best examples of this duality is provided by the class of quasicircles.

Definition 1.1. A Jordan curve $\Gamma$ is a quasicircle if it is of bounded turning; that is, if there exists $R>1$ so that for all points $x, y \in \Gamma$,

$$
\operatorname{diam}\left(\Gamma_{x, y}\right) \leq R|x-y|,
$$

where $\Gamma_{x, y}$ is the subarc of $\Gamma$ connecting $x$ and $y$ which has the smaller diameter.

Loosely speaking, this condition limits "pinching"- a quasicircle cannot visit a point $x$, wander far away, and then return to a point very near $x$. This simple geometric condition

\footnotetext{
* Current address: Department of Mathematics, Texas Tech University, Lubbock, TX 79409-1042, USA. williams@math.ttu.edu.
} 
has powerful analytic consequences. For example, Ahlfors proved that every quasicircle is the image of a circle under a $K$-quasiconformal map of the Riemann sphere $S^{2}$ [1], [2]. (Such a quasicircle would then be called a $K$-quasicircle.) Even more useful is his characterization of quasicircles using reflections and extensions.

Theorem 1.2. If $\Gamma$ is a $K$-quasicircle with complementary components $\Omega$ and $\Omega^{*}$, then the following are equivalent:

1. $\Gamma$ is of $R$-bounded turning.

2. $\Gamma$ is the image of $\partial \mathbb{D}$ under a $K$-quasiconformal map of $S^{2}$.

3. $\Gamma$ admits a $K^{2}$-quasiconformal reflection; that is, there exists a $K^{2}$-quasiconformal involution fixing $\Gamma$ pointwise and interchanging $\Omega$ and $\Omega^{*}$.

4. Every $K_{1}$-quasiconformal map of $\mathbb{D}$ onto $\Omega$ extends to a $K_{1} K^{2}$-quasiconformal map of $S^{2}$ onto itself.

The constants $K$ and $R$ depend only on each other.

Gehring's monograph [14] contains an extensive list of these and other characterizations of quasicircles. The most immediately relevant characterization for us, however, involves the boundary values of Riemann maps [25], [24], [20], [5]. If $f: \mathbb{D} \rightarrow \Omega$ and $g: \mathbb{D}^{*} \rightarrow \Omega^{*}$ are conformal, then their boundary values induce an automorphism $\varphi=f^{-1} g$ of $\partial \mathbb{D}$. Tienari [37] showed that the automorphism induced by Riemann maps from the complementary domains of a quasicircle satisfies the quasisymmetry condition of Beurling and Ahlfors [5], [2].

Definition 1.3. An automorphism $\varphi$ of $\partial \mathbb{D}$ is a $k$-quasisymmetry if for any adjacent subintervals $I$ and $J$ of $\partial \mathbb{D}$ having equal length $|I|_{\mathbb{D}}=|J|_{\mathbb{D}}$, we have

$$
\frac{1}{k} \leq \frac{|\varphi(I)|_{\mathbb{D}}}{|\varphi(J)|_{\mathbb{D}}} \leq k
$$

Theorem 1.4. Let $\Gamma$ be a Jordan curve separating $\mathbb{C}$ into complementary domains $\Omega$ and $\Omega^{*}$, and let $f: \mathbb{D} \rightarrow \Omega$ and $g: \mathbb{D}^{*} \rightarrow \Omega^{*}$ be conformal. Then $\Gamma$ is a quasicircle if and only if the associated automorphism $\varphi=f^{-1} g$ is a quasisymmetry. If $\Gamma$ is normalized so that $0 \in \Omega, 1 \in \Gamma$, and $\infty \in \Omega^{*}$, and if $f$ and $g$ are normalized so that $f(0)=0$, $f(1)=g(1)=1$, and $g(\infty)=\infty$, then $\varphi$ is uniquely determined.

Quasisymmetries are perhaps best known as the boundary values of quasiconformal self-maps of $\mathbb{D}$ [5], [22]; thus the connection to quasicircles is not entirely surprising. A deeper fact is that both the space of normalized quasicircles and the space of normalized quasisymmetries provide models for the Universal Teichmüller Space [23], [13], [24], which parameterizes all conformal structures of all Riemann surfaces.

The Universal Teichmüller Space has gained recent attention by physicists as a setting for string theory [27], [28], [26]. As a closed string propagates through time, it sweeps 
out a Riemann surface. The phase space of the string is then contained in the Universal Teichmüller Space. It may be that switching between models of the Universal Teichmüller Space will be of assistance in string theory computations.

Unfortunately, there is no direct formula for computing a quasisymmetry $\varphi$ from its associated quasicircle $\Gamma$. In this paper we develop a method for approximating $\varphi$ given only $\Gamma$. Our approach uses discrete analytic functions induced by circle packings to approximate the Riemann maps $f$ and $g$.

Approximation Theorem. Given a normalized $K$-quasicircle $\Gamma$ as above, there exists a sequence $\left\{\varphi_{n}\right\}$ of automorphisms of $\partial \mathbb{D}$ constructed using the boundary values of discrete analytic functions and converging uniformly to the normalized quasisymmetry $\varphi=f^{-1} g$ induced by $\Gamma$.

In Section 2 we discuss circle packings and the approximation of conformal maps by packing-induced discrete analytic functions. A combinatorial doubling technique is then used in Section 3 to construct circle packings lying near $\Omega, \Omega^{*}, \mathbb{D}$, and $\mathbb{D}^{*}$. These packings induce discrete maps $f_{n}$ and $g_{n}$ which approximate the Riemann maps $f: \mathbb{D} \rightarrow \Omega$ and $g: \mathbb{D}^{*} \rightarrow \Omega^{*}$. After a few technical issues are resolved, the boundary values of $f_{n}$ and $g_{n}$ can then be used to define approximations $\varphi_{n}$ to $\varphi=f^{-1} g$.

In general, one would expect our sequences $\left\{f_{n}\right\}$ and $\left\{g_{n}\right\}$ to converge uniformly on compact subsets of their domains, but not uniformly on the boundaries of their domains. However, we will show that the ranges of $f_{n}$ and $g_{n}$ are Jordan domains bounded by quasicircles. Theorem 1.2 then provides quasiconformal reflections in these quasicircles. Moreover, as consequence of the combinatorial technique used to construct $f_{n}$ and $g_{n}$, we can also form reflections in the boundaries of the domains of $f_{n}$ and $g_{n}$.

These reflections allow us in Section 4 to extend each $f_{n}$ and $g_{n}$ to (almost) the entire plane. The original boundaries of their domains will then be compact subsets of their new domains, thus allowing us to finesse the question of the convergence of $f_{n}$ and $g_{n}$ on the boundary. As a result, our approximations $\varphi_{n}$ will converge uniformly on $\partial \mathbb{D}$.

Section 5 then completes the proof of our Approximation Theorem, modulo two technical lemmas covered in Section 6.

On a numerical note, circle packing maps are readily computable using Stephenson's CirclePack program [9]. Indeed, many of the figures included herein were generated by CirclePack. Using the new packing algorithm of Collins and Stephenson [7], the radii of the circles can be computed very quickly even for packings with several thousand circles. Unfortunately, beyond He's hexagonal estimate [15], little is known about the rate of convergence of discrete analytic functions to their classical counterparts. For smooth domains, empirical results [9] suggest the convergence might not be as fast as more commonly used methods; however, as observed by Howell and Trefethen [19] and Zemach [39], irregular boundary behavior (such as the type of elongations possible in quasicircles with large $K$ ) often require modification of the standard techniques. It may well be that circle packing methods will prove to have an advantage in such irregular cases. 


\section{Circle Packings and Discrete Analytic Functions}

\subsection{Circle Packings}

We begin by briefly describing circle packings and their powerful connection to geometric function theory. Much more detailed background information is available in the literature [9], [34], [32].

Definition 2.1. A circle packing is a configuration of circles with a specified pattern of tangencies. In particular, if $\mathcal{K}$ is an abstract triangulation, that is, a simplicial 2-complex which is simplicially equivalent to a triangulation of a surface, then a circle packing $P$ for $\mathcal{K}$ is a configuration of circles such that

1. $P$ contains a circle $C_{v}$ for each vertex $v$ in $\mathcal{K}$,

2. $C_{v}$ is externally tangent to $C_{u}$ if $[v, u]$ is an edge of $\mathcal{K}$, and

3. $\left\langle C_{v}, C_{u}, C_{w}\right\rangle$ forms a positively oriented mutually tangent triple of circles if $\langle v, u, w\rangle$ is a positively oriented face of $\mathcal{K}$.

A packing is called univalent if none of its circles overlap, that is, if no pair of circles intersect in more than one point.

Forcing geometric objects to fit together in a predetermined pattern is not always an easy task [12]; indeed, it is not readily apparent that circle packings should exist in any great abundance. Fortunately, circle packings do exist for an incredible variety of complexes [4], [6] and, using Stephenson's CirclePack software, are easy to find [7], [9]. For our purposes, however, we need only the existence of spherical and hexagonal circle packings [3], [21], [35].

Circle Packing Theorem. Given an abstract triangulation $\mathcal{K}$ of a sphere, there exists a univalent circle packing $P$ for $\mathcal{K}$ in $S^{2}$. This packing is unique up to Möbius transformations.

Example 2.2. Observe that euclidean circles of the same size naturally fit together in the constant degree 6 pattern. If we translate, rotate, and scale this pattern so that

1. some circle $C_{v_{0}}$ is centered at 0 ,

2. some neighboring circle of $C_{v_{0}}$ is centered on the real axis, and

3. every circle has radius $1 / 2 n$,

then we form the infinite regular hexagonal packing $H_{n}$. (See Fig. 1.) Notice that we can recover the original degree 6 triangulation by connecting centers of tangent circles by line segments. This embedding of the complex gives a tiling of the plane by equilateral triangles of side length $1 / n$.

Circle packings have been intensely studied because of their deep connections with conformal mapping [8], [18], [29], [36], but many of the properties of packings follow from elementary geometric and combinatorial considerations. One such result is 

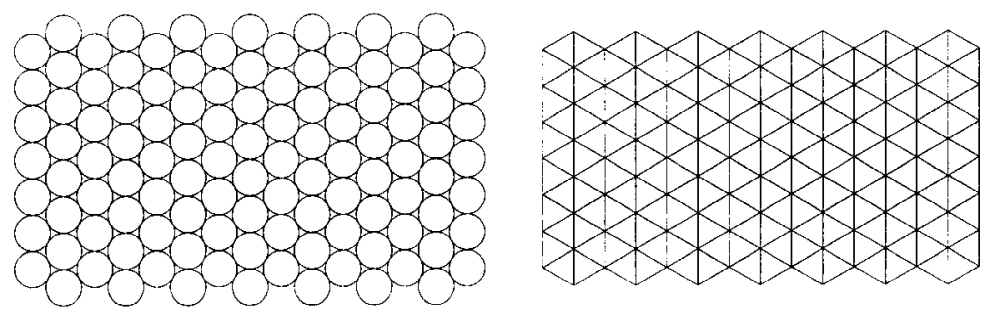

Fig. 1. A portion of the regular hexagonal packing $H_{n}$ (left) and the tiling it induces (right).

the Spherical Length-Area Lemma [38], modeled after Rodin and Sullivan's euclidean Length-Area Lemma [29].

Definition 2.3. A chain of circles in packing $P$ for $\mathcal{K}$ is a collection of circles $C_{v_{1}}, C_{v_{2}}$, $\ldots, C_{v_{n}}$ of $P$ so that $v_{i}$ and $v_{i+1}$ share an edge in $\mathcal{K}, i=1,2, \ldots, n-1$, and $v_{i} \neq v_{j}$, if $i \neq j$. Thus a chain describes a non-self-intersecting edge path in $\mathcal{K}$. A chain is closed if $v_{1}=v_{n}$.

Spherical Length-Area Lemma. Let $P$ be a univalent circle packing on $S^{2}$ and let $C_{v}$ be a circle in $P$ with spherical radius $r$. Assume there exist $m$ closed disjoint chains of circles in $P$ having combinatorial lengths $n_{1}, n_{2}, \ldots, n_{m}$, and each separating $C_{v}$ from two fixed points with spherical distance $\pi / 2 \leq \rho \leq \pi$. Then

$$
r<\frac{2 \pi}{\sqrt{\sum_{i=1}^{m}\left(1 / n_{i}\right)}} .
$$

One particularly useful means of constructing chains is by considering generations of circles about a given vertex. If $v$ is a vertex of $\mathcal{K}$, then the first generation $\mathrm{G}_{\mathcal{K}}(v ; 1)$ consists of all the neighbors of $v$ in $\mathcal{K}$. In general, the $i$ th generation $\mathrm{G}_{\mathcal{K}}(v ; i)$ consists of all vertices which share an edge with a vertex in $\mathrm{G}_{\mathcal{K}}(v ; i-1)$ but which are not contained in $\mathrm{G}_{\mathcal{K}}(v ; i-1)$ or $\mathrm{G}_{\mathcal{K}}(v ; i-2)$. If $v$ is an interior vertex, then for small $i, \mathrm{G}_{\mathcal{K}}(v ; i)$ will correspond to a closed chain.

\subsection{Discrete Analytic Functions}

As described in Example 2.2, a univalent circle packing $P$ produces an embedding of its underlying complex $\mathcal{K}$ when centers of neighboring circles are connected by a geodesic. Indeed, it is the nice properties of such embeddings that provide the nice function-theoretic properties we need.

The region into which $\mathcal{K}$ is thus embedded is called the carrier of $P$, denoted $\operatorname{carr} P$. If $P$ and $\widetilde{P}$ are both univalent packings for the same complex $\mathcal{K}$ and $\operatorname{carr} P, \operatorname{carr} \widetilde{P} \subset \mathbb{C}$, then we may define a map $f: \operatorname{carr} \widetilde{P} \rightarrow \operatorname{carr} P$ as follows: If $\Delta$ and $\widetilde{\Delta}$ are embeddings in carr $P$ and carr $\widetilde{P}$, respectively, of the same face in $\mathcal{K}$, then we map each point $x \in \widetilde{\Delta}$ to the point having the same barycentric coordinates as $x$ in the triangle $\Delta$. The map $f$ is 
then continuous and piecewise affine. Maps induced by circle packings in this way are called discrete analytic functions [9], [34].

A first glimpse of the deep connection between these discrete analytic functions and classical geometric function theory is provided by the Ring Lemma of Rodin and Sullivan [29].

Ring Lemma. If $P$ is a univalent circle packing for $\mathcal{K}$ and the degree of $\mathcal{K}$ is bounded by $M<\infty$, then the angles in triangles in $\operatorname{carr} P$ which do not contain a boundary vertex are bounded away from 0 and $\pi$. Moreover, this bound depends only $M$.

It is easy to show that if $f$ is an affine map from some euclidean triangle $\widetilde{\Delta}$ to a euclidean triangle $\Delta$, then $f$ is $K$-quasiconformal, where $K$ depends only on the maximum difference between angles in $\widetilde{\Delta}$ and corresponding angles in $\Delta$. Consequently, discrete analytic functions are quasiconformal, if the degree of $\mathcal{K}$ is bounded.

Corollary 2.4. If $P$ and $\widetilde{P}$ are two univalent packings in $\mathbb{C}$ for the same complex $\mathcal{K}$, with $\operatorname{deg} \mathcal{K}<\infty$, then the induced discrete analytic function $f: \operatorname{carr}(\widetilde{P}) \rightarrow \operatorname{carr}(P)$ is $K$-quasiconformal on faces which do not contain a boundary vertex. Moreover, $K$ depends only on the degree of $\mathcal{K}$.

Thus far we have considered only the effects of the local combinatorics on faces. However, if a face is "deep inside" the carrier, the global combinatorics can exert a powerful influence [29].

Hex Packing Lemma. If a circle $C_{v}$ has six neighbors and is surrounded by n univalent generations of degree 6 circles in a euclidean circle packing, then the ratio between the radius of $C_{v}$ and the radius of any neighboring circle $C_{u}$ is bounded above and below by constants which depend only on $n$ and decrease to 1 as $n \rightarrow \infty$.

At first, the degree 6 condition seems rather odd. However, as illustrated in Example 2.2, circles of the same size naturally fit together in the constant degree 6 pattern. In fact, the only univalent packings for this infinite degree 6 pattern are the regular hexagonal packings in which each circle has the same size [30], [29]. Thus the Hex Packing Lemma asserts that if a packing shares the combinatorics of $H_{n}$ near $C_{v}$, it must possess nearly the same geometry at $C_{v}$. In particular, deep inside a finite degree 6 packing, triangles of the carrier will not only have bounded angles, but will in fact be nearly equilateral. Thus discrete analytic functions are almost analytic (1-quasiconformal) deep inside a degree 6 packing.

Corollary 2.5. If a degree 6 vertex $v$ in $\mathcal{K}$ is surrounded by $n$ generations of degree 6 vertices, and $f: \operatorname{carr} \widetilde{P} \rightarrow \operatorname{carr} P$ is a discrete analytic function between univalent packings for $\mathcal{K}$, then the dilatation of $f$ on faces containing $v$ decreases to 1 as $n \rightarrow \infty$.

These corollaries were used by Rodin and Sullivan [29] to prove a circle packing version of the Riemann Mapping Theorem. If $\Omega \subsetneq \mathbb{C}$ and $p, q \in \Omega$, then the Riemann Mapping Theorem promises a unique conformal map $f: \mathbb{D} \rightarrow \Omega$ normalized so that 
$f(0)=p$ and $f^{-1}(q)>0$. Rodin and Sullivan constructed a sequence of discrete analytic functions which converge locally uniformly to $f$. More precisely, if $H_{n}$ is the regular hexagonal packing described in Example 2.2, then the portion of $H_{n}$ intersecting $\Omega$ and containing $p$ gives a packing $P_{n}$ in $\Omega$. A corollary of the Circle Packing Theorem ensures the existence of an appropriately normalized packing $\widetilde{P}_{n}$ in $\mathbb{D}$ with the same underlying complex as $P_{n}$.

Rodin-Sullivan Theorem. The packings $P_{n}$ and $\widetilde{P}_{n}$ described above induce discrete analytic functions $f_{n}$ : carr $\widetilde{P}_{n} \rightarrow \operatorname{carr} P_{n}$ which converge uniformly on compact subsets of $\mathbb{D}$ to the Riemann map $f$ as $n \rightarrow \infty$.

Remark 2.6. It should be noted that the Rodin-Sullivan Theorem holds for much more general packings [17], [18], [33], [31], [16]. However, to simplify our arguments as much as possible we limit ourselves to hexagonal combinatorics.

Remark 2.7. We also mention that while the use of circle packings to create discrete analytic functions began with Thurston [36], a related notion was already present in the works of Duffin [10], [11].

\section{Construction of the Approximating Maps}

As indicated in Section 2, circle packing provides a discrete approach to conformal mapping problems. We now use this method to approximate not just conformal maps themselves, but their boundary values as well.

\subsection{Construction of Packings in $\Omega$ and $\Omega^{*}$}

Fix a $K$-quasicircle $\Gamma$ with complementary domains $\Omega$ and $\Omega^{*}$. Following the usual normalization, we assume $1 \in \Gamma, 0 \in \Omega$, and $\infty \in \Omega^{*}$.

Now consider the subcomplex formed by deleting from the carrier of $H_{n}$ all triangles which hit $\Gamma$ or $\Omega^{*}$. To avoid certain technical difficulties, we further delete any face which does not contain an interior vertex. Note that if $u$ is an interior vertex, then no faces containing $u$ are deleted, so $u$ remains an interior vertex. Thus no new boundary vertices are formed by this trimming.

In general, this complex may have many components, but we consider only the component $\mathcal{K}_{n}$ containing $v_{0}$. (Recall that $H_{n}$, and consequently now $\mathcal{K}_{n}$, were normalized so that $v_{0}$ corresponds to the point 0 .) See Fig. 2.

We denote the packing formed by the portion of $H_{n}$ corresponding to $\mathcal{K}_{n}$ by $P_{n}$. Note that $\Omega_{n}=\operatorname{carr} P_{n}$ lies entirely in $\Omega$. Moreover, both $\Omega_{n}$ and $\mathcal{K}_{n}$ are simply connected since "holes" in either would imply the existence of "holes" in the (simply connected) Jordan domain $\Omega$.

We are not overly concerned with the distinction between $\mathcal{K}_{n}$ and the embedding for it provided by $\Omega_{n}$. In particular, we refer to points in $\partial \Omega_{n}$ corresponding to boundary vertices of $\mathcal{K}_{n}$ as "boundary vertices of $\Omega_{n}$." 

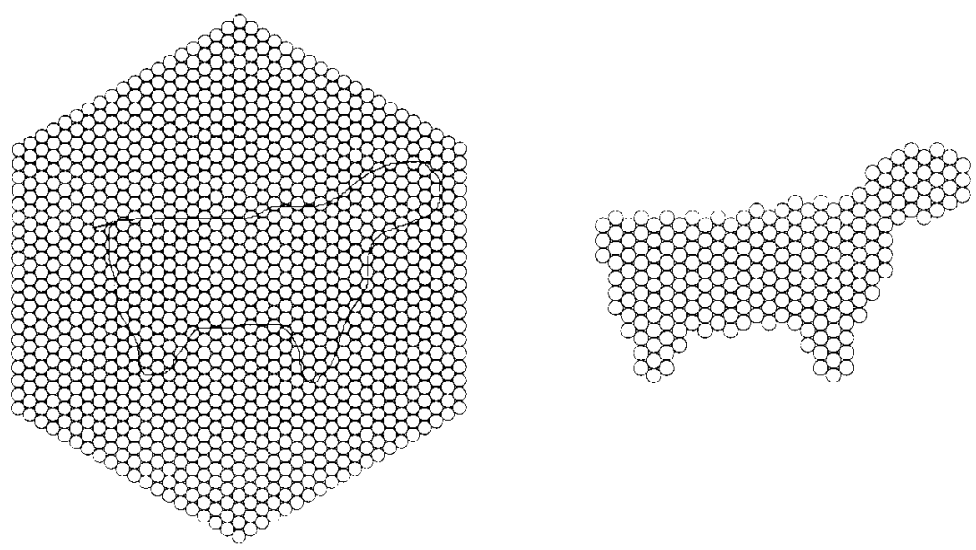

Fig. 2. A cow-shaped quasicircle. A cow-shaped quasicircle $\Gamma$ and a portion of $H_{n}$ before and after trimming.

Note that every boundary vertex of $\Omega_{n}$ is at most one triangle away from $\Gamma$. That is, every boundary vertex $v$ must have been a vertex of one of the triangles in $\operatorname{carr} H_{n}$ which was removed to form $\mathcal{K}_{n}$. Thus $\Gamma$ must hit an equilateral triangle $\Delta_{v}$ of side length $1 / n$ of which $v$ is a vertex. Hence there is a line segment $\gamma_{v}$ connecting $v$ to $\Gamma$ having length at most $1 / n$ and lying either on or inside $\Delta_{v}$. Moreover, we may choose $\gamma_{v}$ short enough so that $\gamma_{v} \cap \Gamma$ is a single point $\Gamma_{v}$. See Fig. 3.

A similar construction can be used to build a sequence of degree 6 packings contained in $\Omega^{*}$. Unfortunately, since $H_{n}$ accumulates at $\infty \in \Omega^{*}$, we cannot use $H_{n}$ directly. Instead, we stereographically project each $H_{n}$ to $S^{2}$, apply a Möbius rotation of $S^{2}$ fixing

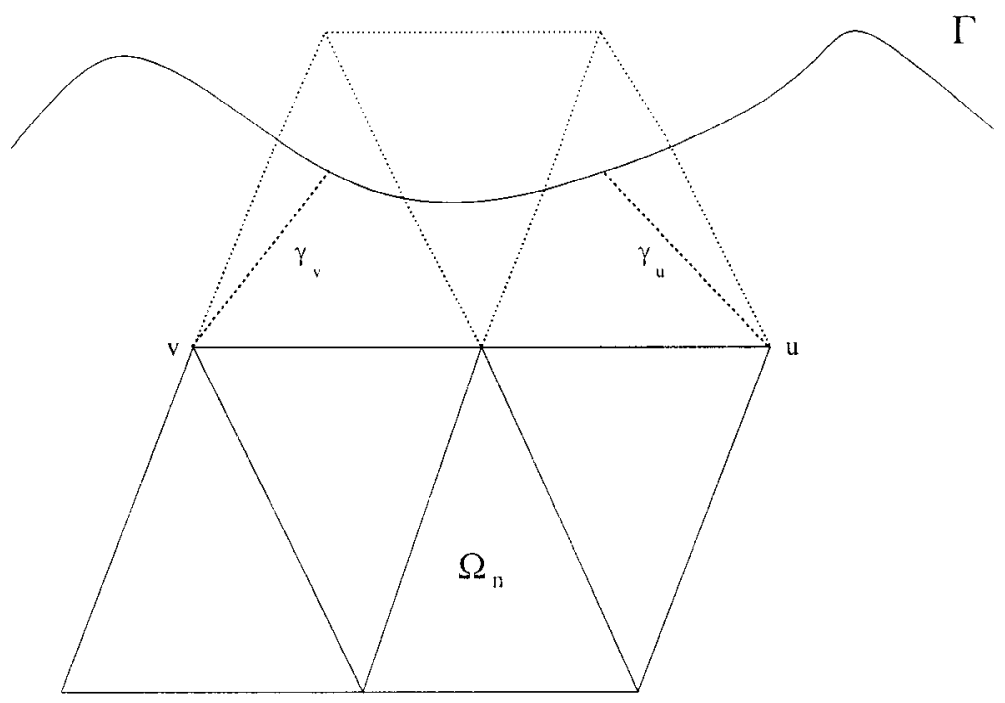

Fig. 3. The curve $\Gamma$ and the region $\Omega_{n}$. Several deleted triangles are shown using dotted lines along with line segments $\gamma_{v}$ and $\gamma_{u}$ joining $\partial \Omega_{n}$ to $\Gamma$. 
1 and interchanging 0 and $\infty$, and then stereographically project the resulting packing back to $\mathbb{C}$. Each of these new euclidean packings $H_{n}^{*}$ will now accumulate only at 0 ; hence, only finitely many circles will lie in $\Omega^{*}$. Mimicking our earlier construction, we form $P_{n}^{*}$ by first removing from $H_{n}^{*}$ any faces which hit $\Omega$ or $\Gamma$ and then trimming extraneous vertices as before. However, unlike before, the radii of circles in $P_{n}^{*}$ now vary within the packing, but since the maximum radius of boundary circles does decrease to 0 as $n \rightarrow \infty$, we can renumber so that the radii of boundary circles in $P_{n}^{*}$ are uniformly small. In particular, we may assume each vertex $w$ on the boundary of $\Omega_{n}^{*}=\operatorname{carr} P_{n}^{*}$ is connected to $\Gamma$ by a line segment $\gamma_{w}$ lying outside $\Omega_{n}^{*}$ and having length at most $1 / n$.

Note that one circle $C_{w_{\infty}}$ of $P_{n}^{*}$ will be centered at $\infty$ so that the underlying complex $\mathcal{K}_{n}^{*}$ for $P_{n}^{*}$ is not embedded in $\mathbb{C}$, but in $C \cup\{\infty\}$. We denote the unbounded open region corresponding to the open star of $w_{\infty}$ by $S_{P_{n}^{*}}\left(w_{\infty}\right)$. This minor annoyance will complicate our notation, but will not fundamentally alter our program.

Now observe that, as $n \rightarrow \infty$,

$$
\begin{array}{rlrl}
\Omega_{n} & \rightarrow \Omega, & \Omega_{n}^{*} \backslash S_{P_{n}^{*}}\left(w_{\infty}\right) & \rightarrow \Omega^{*}, \\
\partial \Omega_{n} & \rightarrow \Gamma, & \partial \Omega_{n}^{*} \rightarrow \Gamma .
\end{array}
$$

In fact, the geometry of $\Gamma$ and the combinatorial structure of $H_{n}$ allow us to estimate the proximity of $\partial \Omega_{n}$ and $\partial \Omega_{n}^{*}$ to $\Gamma$.

Proximity Lemma. Using the notation established above, for large $n$ there exist homeomorphisms $l_{n}: \partial \Omega_{n} \rightarrow \Gamma$ and $u_{n}: \partial \Omega_{n}^{*} \rightarrow \Gamma$ such that for all $z \in \partial \Omega_{n}$ and $w \in \partial \Omega_{n}^{*}$,

$$
\begin{aligned}
\left|l_{n}(z)-z\right| & \leq \frac{M}{2 n}, \\
\left|u_{n}(w)-w\right| & \leq \frac{M}{2 n},
\end{aligned}
$$

where $M$ depends only on $K$. In particular, we have

$$
\left|l_{n}^{-1} u_{n}(w)-w\right| \leq \frac{M}{n} .
$$

Since $\partial \Omega_{n}$ and $\partial \Omega_{n}^{*}$ lie so near the $K$-quasicircle $\Gamma$, we would hope $\partial \Omega_{n}$ and $\partial \Omega_{n}^{*}$ are quasicircles themselves. The Extension Lemma shows this is in fact the case.

Extension Lemma. For each $n, \partial \Omega_{n}$ and $\partial \Omega_{n}^{*}$ are $K^{\prime}$-quasicircles, where $K^{\prime}$ depends only on $K$. In particular, there exist $\left(K^{\prime}\right)^{2}$-quasiconformal reflections $J_{n}$ and $J_{n}^{*}$ in $\partial \Omega_{n}$ and $\partial \Omega_{n}^{*}$, respectively.

As the proofs are extremely technical and the conclusions of the lemmas rather easy to believe, we defer the details to Section 6.

Remark 3.1. Although the constants in the Proximity Lemma are tied to the hexagonal combinatorics of $H_{n}$, a similar result holds for other packings. Indeed, the entire approximation scheme described herein can be performed using any sequence of packings having uniformly bounded degree and mesh decreasing to 0 . 


\subsection{Construction of Packings in $\mathbb{D}$ and $\mathbb{D}^{*}$}

Now we wish to construct a circle packing lying near $\mathbb{D}$ for each $\mathcal{K}_{n}$ and a packing in $\mathbb{D}^{*}$ for each $\mathcal{K}_{n}^{*}$. Such a packing for $\mathcal{K}_{n}$ is usually constructed by adding a single vertex with edges to each boundary vertex. The resulting spherical triangulation has a packing on $S^{2}$ by the Circle Packing Theorem. We may normalize this packing so that the circle corresponding to the added vertex corresponds to $\partial \mathbb{D}$ and the original boundary vertices of $\mathcal{K}_{n}$ correspond to circles in $\mathbb{D}$ tangent to $\partial \mathbb{D}$. Stereographic projection leaves $\partial \mathbb{D}$ unchanged, thus providing a euclidean packing for $\mathcal{K}_{n}$ in $\mathbb{D}$.

Unfortunately, this construction is insufficient for our needs. Instead, we use a combinatorial "doubling trick." To form the combinatorial double $\mathcal{K}^{2}$ of a complex $\mathcal{K}$, we construct another copy $\widehat{\mathcal{K}}$ of $\mathcal{K}$ with the opposite orientation and then identify boundary edges of $\mathcal{K}$ and $\widehat{\mathcal{K}}$ which are copies of one another.

In general the double of an abstract triangulation need not be an abstract triangulation. For example, a boundary vertex with no interior neighbors will have degree 2 in the combinatorial double. Consequently, such a double could not be simplicially equivalent to a triangulation of a surface. Recall, however, that we trimmed from each $\mathcal{K}_{n}$ any boundary vertices without an interior neighbor. This trimming is sufficient to ensure that $\mathcal{K}_{n}^{2}$ is indeed an abstract triangulation. Since $\mathcal{K}_{n}$ was simplicially equivalent to a closed disk, $\mathcal{K}_{n}^{2}$ will be an abstract triangulation of a sphere.

Now the Circle Packing Theorem gives a circle packing $\widetilde{P}_{n}$ on $S^{2}$ for $\mathcal{K}_{n}^{2}$. We may normalize so that the circle for $v_{0}$ is centered at the origin, the circle for the doubled copy $\widehat{v}_{0}$ of $v_{0}$ is centered at infinity, and the point corresponding to $l_{n}^{-1}(1)$ is located at 1. That is, since $1 \in \Gamma$, the map $l_{n}^{-1}$ promised by the Proximity Lemma will send 1 to some point on $\partial \Omega_{n}$. However, $\partial \Omega_{n}$ is a polygon consisting of line segments forming the embedded images of boundary edges of $\mathcal{K}_{n}$. Thus $l_{n}^{-1}(1)$ lies on some line segment $\overline{a b}$ corresponding to an edge $\left[u_{a}, u_{b}\right]$ of $\mathcal{K}_{n}$. In particular,

$$
l_{n}^{-1}(1)=(1-t) a+t b,
$$

for some $0 \leq t<1$. Now if $\tilde{a}$ and $\underset{\widetilde{b}}{\widetilde{P}}$ are the euclidean centers of the circles $C_{u_{a}}$ and $C_{u_{b}}$, respectively, in the new packing $\widetilde{P}_{n}$, then we normalize $\widetilde{P}_{n}$ so that

$$
(1-t) \tilde{a}+t \tilde{b}=1 .
$$

The affine nature of this normalization is of course motivated by the piecewise affine definition of discrete analytic functions.

Note that $\widetilde{P}_{n}$ must be symmetric about $\partial \mathbb{D}$, the equator of $S^{2}$, for if not, the Möbius rotation of $S^{2}$ which fixes 1 and interchanges 0 and $\infty$ would result in a different packing with the same combinatorics and normalization, violating the uniqueness portion of the Circle Packing Theorem. In particular, this symmetry implies that circles corresponding to boundary vertices of $\mathcal{K}_{n}$ are all centered on $\partial \mathbb{D}$. In fact, $\widetilde{P}_{n}$ provides an embedding of $\mathcal{K}_{n}$ in the lower hemisphere of $S^{2}$ and an embedding of $\widehat{\mathcal{K}}_{n}$ in the upper hemisphere. Unfortunately, this embedding uses spherical triangles, not the euclidean triangles needed to construct our discrete analytic functions. See Fig. 4.

To arrive at a euclidean embedding, we stereographically project to the plane the portion of $\widetilde{P}_{n}$ corresponding to $\mathcal{K}_{n}$. The euclidean carrier $D_{n}$ of this packing can then be used as the domain for a discrete analytic function. 


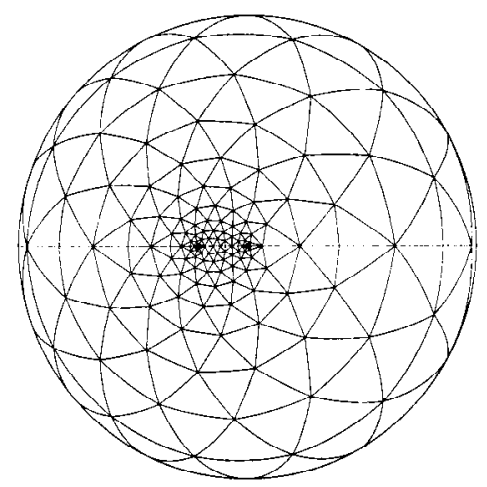

Fig. 4. A doubled complex. The doubled complex on $S^{2}$ for the $\mathcal{K}_{n}$ depicted in Fig. 2.

Because the density of the spherical metric is higher in $\mathbb{D}$ than in $\mathbb{D}^{*}, \partial D_{n}$ is a polygon circumscribing $\mathbb{D}$, not $\partial \mathbb{D}$ itself. See Fig. 5. However, since the spherical carrier was precisely equal to $\mathbb{D}$, the switching of geometries induces a map

$$
p_{n}: \partial \mathbb{D} \rightarrow \partial D_{n}
$$

by mapping the spherical centers of the circles centered on $\partial \mathbb{D}$ to the euclidean centers of the stereographic projection of these circles and then extending affinely on the edges.

In a similar way, we can double the complex $\mathcal{K}_{n}^{*}$ used to define $\Omega_{n}^{*}$ in Section 3.1 and pack the resulting complex on $S^{2}$. We normalize this packing $\widetilde{P}_{n}^{*}$ so that $C_{w_{\infty}}$ is centered at $\infty, C_{\hat{w}_{\infty}}$ is centered at 0 (where $\widehat{w}_{\infty}$ is the copy of $w_{\infty}$ in $\widehat{\mathcal{K}_{n}^{*}}$ ), and the point corresponding to $u_{n}^{-1}(1)$ is centered at 1 . Again we stereographically project the portion
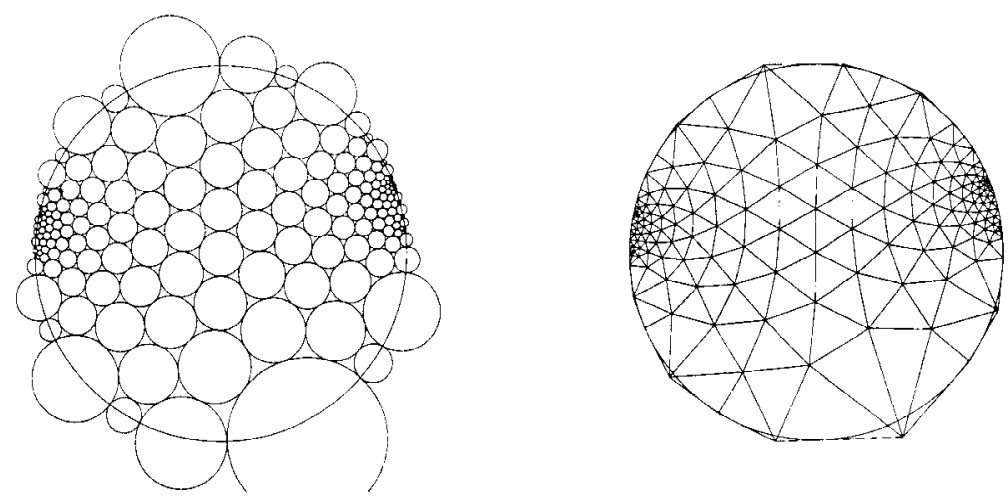

Fig. 5. A euclidean complex. The packing (left) of Fig. 4 stereographically projected to a neighborhood of $\mathbb{D}$. Note the centers of boundary circles lie outside $\mathbb{D}$. The triangles of the carrier $D_{n}$ (right) extending outside $\mathbb{D}$ are clearly visible. 
corresponding to $\mathcal{K}_{n}^{*}$ to $\mathbb{C}$ and denote its euclidean carrier by $D_{n}^{*}$. As before, we obtain the piecewise-defined map

$$
p_{n}^{*}: \partial \mathbb{D} \rightarrow \partial D_{n}^{*}
$$

Observe that in $\widetilde{P}_{n}^{*}$ as well in as $P_{n}^{*}$, the circle $C_{w_{\infty}}$ is centered at $\infty$. Thus the stereographic projection of $P_{n}^{*}$ to $\mathbb{C}$ does not provide a proper embedding of $\mathcal{K}_{n}^{*}$ in $\mathbb{C}$. Following our earlier notation, we denote the unbounded open region corresponding to the open star of $w_{\infty}$ in $\widetilde{P}_{n}^{*}$ by $S_{\tilde{P}_{n}^{*}}\left(w_{\infty}\right)$.

\subsection{Construction of the Approximating Maps}

At last we can define our approximating maps. The packings constructed above induce discrete analytic functions

$$
\begin{aligned}
& f_{n}: D_{n} \rightarrow \Omega_{n}, \\
& g_{n}: D_{n}^{*} \backslash S_{\tilde{P}_{n}^{*}}\left(w_{\infty}\right) \rightarrow \Omega_{n}^{*} \backslash S_{P_{n}^{*}}\left(w_{\infty}\right) .
\end{aligned}
$$

We now use the boundary values of these discrete analytic functions to define our approximations

$$
\varphi_{n}=p_{n}^{-1} \circ f_{n}^{-1} \circ l_{n}^{-1} \circ u_{n} \circ g_{n} \circ p_{n}^{*}
$$

to the quasisymmetry $\varphi$ induced by $\Gamma$.

Lest we lose sight of the simple geometry in this maze of compositions, we recall what $\varphi_{n}$ actually does. First $\varphi_{n}$ maps the boundary of $\mathbb{D}$ to the boundary of the carrier of a euclidean circle packing. This is merely a technical correction to allow us to define $\varphi_{n}$ on $\partial \mathbb{D}$ itself rather than on a polygon about $\partial \mathbb{D}$. Then we move to another circle packing for the same complex $\mathcal{K}_{n}^{*}$, but this one repacked to lie inside $\Omega^{*}$. Next we "jump across" $\Gamma$ to the carrier of a packing lying in $\Omega$. Another repacking takes us back to a polygon about $\partial \mathbb{D}$, and a final technical correction moves us to $\partial \mathbb{D}$. The unfortunate technical considerations aside, this is precisely a discrete version of $f^{-1} g$.

Note that the boundary vertices of $D_{n}$ and $D_{n}^{*}$ are actually interior vertices of the carriers of the stereographic projections of the packings $\widetilde{P}_{n}$ and $\widetilde{P}_{n}^{*}$, respectively. Moreover, the boundary vertices of $\Omega_{n}$ and $\Omega_{n}^{*}$ are also interior vertices of the carriers of the packings $H_{n}$ and $H_{n}^{*}$, respectively. Thus since the degrees of $\mathcal{K}_{n}$ and $\mathcal{K}_{n}^{*}$ are at most 6, Corollary 2.4 implies that the families $\left\{f_{n}\right\}$ and $\left\{g_{n}\right\}$ are uniformly $K_{1}$ quasiconformal.

\section{Combinatorial Reflections}

Recall that the Rodin-Sullivan Theorem promised the convergence of discrete analytic functions to the Riemann map uniformly on compact subsets of $\mathbb{D}$, not necessarily on $\overline{\mathbb{D}}$. In general, there is no reason to expect the boundary values of the discrete analytic functions to converge at all. However, if we could extend these maps to a neighborhood of $\partial \mathbb{D}$ while maintaining the locally uniform convergence, then we would finesse the question of convergence on the boundary.

Thus the key to convergence is extension, and the key to extension is reflection. 


\subsection{Combinatorial Reflections}

Recall that constructing $\widetilde{P}_{n}$ by doubling provided a packing for $\mathcal{K}_{n}$ in the lower hemisphere of $S^{2}$ and a packing for $\widehat{\mathcal{K}}_{n}$ in the upper hemisphere. Since $\widehat{\mathcal{K}}_{n}$ is simply a copy of $\mathcal{K}_{n}$ with reversed orientation, we would like to construct a piecewise affine "discrete reflection" interchanging $D_{n}$ and the carrier $\widehat{D}_{n}$ of the stereographic projection of the packing for $\widehat{\mathcal{K}}_{n}$.

Unfortunately, the circle for the vertex $\widehat{v}_{0}$ of $\widehat{\mathcal{K}}_{n}$ is centered at $\infty$. Thus $\widehat{\mathcal{K}}_{n}$ will be embedded not in $\mathbb{C}$, but in $\mathbb{C} \cup\{\infty\}$. Faces of $\widehat{\mathcal{K}}_{n}$ containing $\widehat{v}_{0}$ will not be realized as triangles lying completely in $\mathbb{C}$ and thus cannot be used to construct the discrete reflection. We denote the open unbounded region corresponding to the open star of $\widehat{v}_{0}$ by $S_{\tilde{P}_{n}}\left(\widehat{v}_{0}\right)$. Similarly, we denote the open subset of $D_{n}$ corresponding to the open star of $v_{0}$ by $S_{\tilde{P}_{n}}\left(v_{0}\right)$.

Lemma 4.1. There exists a discrete reflection $\iota_{n}$ in $\partial D_{n}$ interchanging $D_{n} \backslash S_{\tilde{P}_{n}}\left(v_{0}\right)$ with $\widehat{D}_{n} \backslash S_{\tilde{P}_{n}}\left(\widehat{v}_{0}\right)$. Similarly, there exists a discrete reflection $\imath_{n}^{*}$ interchanging $D_{n}^{*} \backslash S_{\tilde{P}_{n}^{*}}\left(w_{\infty}\right)$ with $\widehat{D_{n}^{*}} \backslash S_{\tilde{P}_{n}^{*}}\left(\widehat{w}_{\infty}\right)$, where $\widehat{D_{n}^{*}}$ is, of course, the carrier of the stereographic projection of the portion of $\widetilde{P}_{n}^{*}$ corresponding to $\widehat{\mathcal{K}}_{n}^{*}$. Moreover, $\imath_{n}$ and $\imath_{n}^{*}$ are both $K_{2}$-quasiconformal, where $K_{2}$ is independent of $n$.

Proof. Since $D_{n} \backslash S_{\tilde{P}_{n}}\left(v_{0}\right)$ and $\widehat{D}_{n} \backslash S_{\tilde{P}_{n}}\left(\widehat{v}_{0}\right)$ consist of euclidean triangles in the same combinatorial pattern, we can define our reflection in the same way we defined discrete analytic functions. That is, each face embedded in $D_{n} \backslash S_{\tilde{P}_{n}}\left(v_{0}\right)$ can be mapped to its combinatorial copy in $\widehat{D}_{n} \backslash S_{\tilde{P}_{n}}\left(\widehat{v}_{0}\right)$ by an (orientation-reversing) affine map. Taken together, we have an (orientation-reversing) piecewise affine map $i_{n}$ interchanging $D_{n} \backslash S_{\tilde{P}_{n}}\left(v_{0}\right)$ with $\widehat{D}_{n} \backslash S_{\tilde{P}_{n}}\left(\widehat{v}_{0}\right)$ and fixing $\partial D_{n}$ pointwise.

The construction of $l_{n}^{*}$ is, of course, similar.

Recall that stereographic projection of the packings $\widetilde{P}_{n}$ and $\widetilde{P}_{n}^{*}$ had no boundary circles. Thus by the comments preceding Corollary $2.4, \iota_{n}$ and $\imath_{n}^{*}$ are $K_{2}$-quasiconformal, where $K_{2}$ depends only on the degree of the combinatorial doubles of $\mathcal{K}_{n}$ and $\mathcal{K}_{n}^{*}$. However, these $\mathcal{K}_{n}$ and $\mathcal{K}_{n}^{*}$ were formed from degree 6 complexes; hence their doubles can have degree at most 12 . Consequently $K_{2}$ is independent of $n$.

Note that unlike the quasiconformal reflections described in Theorem 1.2, these reflections are purely combinatorial in nature, arising from our doubling construction rather than the geometry of $\partial D_{n}$ or $\partial D_{n}^{*}$.

\subsection{Shrinking the Exceptional Sets}

The need to consider the exceptional sets $S_{\tilde{P}_{n}}\left(v_{0}\right), S_{\tilde{P}_{n}}\left(\widehat{v}_{0}\right), S_{\tilde{P}_{n}^{*}}\left(w_{\infty}\right)$, and $S_{\tilde{P}_{n}^{*}}\left(\widehat{w}_{\infty}\right)$ arose only from our normalization placing $w_{\infty}$ and $\widehat{v}_{0}$ at $\infty$. Such technical considerations far from the boundary should have little impact on the boundary behavior of $f_{n}$ and $g_{n}$. Indeed, these exceptional sets will completely disappear in the limit. 
Lemma 4.2. The spherical diameters of $S_{\tilde{P}_{n}}\left(v_{0}\right), S_{\tilde{P}_{n}}\left(\widehat{v}_{0}\right), S_{\tilde{P}_{n}^{*}}\left(w_{\infty}\right)$, and $S_{\tilde{P}_{n}^{*}}\left(\widehat{w}_{\infty}\right)$ decrease to 0 as $n \rightarrow \infty$.

Proof. It suffices to show that the spherical radii of the circles in $\widetilde{P}_{n}$ and $\widetilde{P}_{n}^{*}$ corresponding to $v_{0}, \widehat{v}_{0}, w_{\infty}, \widehat{w}_{\infty}$, and their neighbors decrease to 0 . Note that as $n \rightarrow \infty$, the number $m$ of generations of degree 6 vertices surrounding $w_{\infty}$ and each of its neighbors in $\mathcal{K}_{n}^{*}$ increases without bound. Each generation $\mathrm{G}_{\mathcal{K}_{n}}\left(w_{\infty} ; i\right)$ forms a chain of length $6 i$. Hence by the Spherical Length-Area Lemma, the spherical radius of $C_{w_{\infty}}$ and each of its neighbors is bounded by

$$
\frac{2 \pi}{\sqrt{\sum_{i=1}^{m}(1 / 6 i)}},
$$

with $m \rightarrow \infty$ as $n \rightarrow \infty$. Since $\sum_{i=1}^{\infty}(1 / 6 i)$ diverges, the spherical diameter of $S_{\tilde{P}_{n}^{*}}\left(w_{\infty}\right)$ decreases to 0 as $n \rightarrow \infty$. Of course, the same holds for $S_{\tilde{P}_{n}}\left(v_{0}\right), S_{\tilde{P}_{n}}\left(\widehat{v}_{0}\right)$, and $S_{\tilde{P}_{n}^{*}}\left(\widehat{w}_{\infty}\right)$ as well.

\subsection{Extension of the Discrete Maps}

Now by the Extension Lemma we can extend each $f_{n}$ and $g_{n}$ by setting

$$
\begin{array}{ll}
f_{n}=J_{n} \circ f_{n} \circ l_{n} & \text { on } \widehat{D}_{n} \backslash S_{\tilde{P}_{n}}\left(\widehat{v}_{0}\right), \\
g_{n}=J_{n}^{*} \circ g_{n} \circ l_{n}^{*} & \text { on } \widehat{D}_{n}^{*} \backslash S_{\tilde{P}_{n}^{*}}\left(\widehat{w}_{\infty}\right) .
\end{array}
$$

Note that each extended $f_{n}$ and $g_{n}$ is then $K=K_{1} K_{2}\left(K^{\prime}\right)^{2}$-quasiconformal. Moreover, Lemma 4.2 implies that on each compact subset of $\mathbb{C}, g_{n}$ and $f_{n}$ are defined for sufficiently large $n$.

\section{Proof Modulo the Extension Lemma}

\subsection{Convergence of $p_{n}$ and $p_{n}^{*}$}

The convergence of $p_{n}$ and $p_{n}^{*}$ to the identity map follows directly from the Spherical Length-Area Lemma.

Lemma 5.1. As $n \rightarrow \infty$, the radii of circles in $\widetilde{P}_{n}$ and $\widetilde{P}_{n}^{*}$ corresponding to boundary vertices of $\mathcal{K}_{n}$ and $\mathcal{K}_{n}^{*}$ decrease uniformly to 0 . Consequently both $p_{n}$ and $p_{n}^{*}$ converge uniformly to the identity map on $\partial \mathbb{D}$ as $n \rightarrow \infty$.

Proof. Since $\Gamma$ is compact and misses 0 , there is a lower bound $d$ on the distance of points on $\Gamma$ from 0 . By our construction, the distance of points on $\partial \Omega_{n}$ from 0 is greater than $d-2 / n$. Thus each boundary circle of $\Omega_{n}$ is separated in the packing $H_{n}$ from $v_{0}$ by at least

$$
\left\lfloor\frac{d-2 / n}{2 / n}\right\rfloor=\left\lfloor\frac{n d-2}{2}\right\rfloor
$$


generations of circles. That is, there are at least $\lfloor(n d-2) / 2\rfloor$ chains of circles in $H_{n}$ about each boundary circle separating it from 0 . However, recall that in $H_{n}$ the $i$ th such chain has length $6 i$.

Now when $H_{n}$ is intersected with the inside of $\Gamma$, these chains become half-chains in $\Omega_{n}$. Thus in the spherical packing for the combinatorial double of $\Omega_{n}$ constructed above, each circle on the original boundary of $\Omega_{n}$ is surrounded by $\lfloor(n d-2) / 2\rfloor$ chains of circles separating it from both 0 and $\infty$. Note that the $i$ th chain in the doubled complex now has length at most $12 i$. Thus by the Spherical Length-Area Lemma, the spherical radius of any circle corresponding to a vertex on $\partial D_{n}$ is less than

$$
\frac{2 \pi}{\sqrt{\sum_{i=1}^{n}(1 / 12 i)}} \rightarrow 0 .
$$

Since the euclidean projection of these circles are bounded away from $\infty$, their euclidean radii must be uniformly comparable to their spherical radii. Thus the euclidean radii of circles on $\partial \Omega_{n}$ must decrease uniformly to 0 . It is easy to see in this case that both $p_{n}$ and $p_{n}^{*}$ converge uniformly to the identity map on $\partial \mathbb{D}$ as $n \rightarrow \infty$.

\subsection{Proof of Approximation Theorem}

Assuming the Proximity Lemma and the Extension Lemma, the proof of our Approximation Theorem follows easily.

Approximation Theorem. Given a normalized $K$-quasicircle $\Gamma$ as above, there exists a sequence $\left\{\varphi_{n}\right\}$ of automorphisms of $\partial \mathbb{D}$ constructed using the boundary values of discrete analytic functions and converging uniformly to the normalized quasisymmetry $\varphi=f^{-1} g$ induced by $\Gamma$.

Proof. First note each $f_{n}$ is $K_{1}$-quasiconformal and omits $\infty$ while fixing both 0 and 1. Thus by a standard normal family argument [25], a subsequence of $\left\{f_{n}\right\}$ converges uniformly on compact subsets of $\mathbb{C}$ to a $K_{1}$-quasiconformal homeomorphism $f$. Similarly each $g_{n}$ omits both 0 and $\infty$ while fixing 1. A subsequence of $\left\{g_{n}\right\}$ will then converge uniformly on compact subsets of $\mathbb{C}$ to $K_{1}$-quasiconformal map $g$. In general, a sequence which fixes only one point may converge to either a homeomorphism or a constant map; however, Lemma 4.2 ensures that $g$ is not constant.

Now, the proof of Lemma 4.2 can be used to show that by taking $n$ sufficiently large, any compact subset of $\mathbb{D}$ can be separated from $\partial \mathbb{D}$ by arbitrarily many generations of degree 6 vertices in $D_{n}$. Thus by the Hex Packing Lemma, the dilatation of $f_{n}$ on any compact subset of $\mathbb{D}$ can be made arbitrarily close to 1 by taking $n$ large. Hence the limit map $f$ must be conformal on $\mathbb{D}$, and similarly $g$ must be conformal on $\mathbb{D}^{*}$.

Note that $f$ and $g$ satisfy the normalization $f(0)=0, g(\infty)=\infty$, and $f(1)=$ $g(1)=1$. Furthermore, any subsequences of $\left\{f_{n}\right\}$ and $\left\{g_{n}\right\}$ would again be normal and have further subsequences converging to conformal maps satisfying the same normalization as $f$ and $g$. However, Riemann maps so normalized are unique. Thus it follows 
that not just subsequences, but the entire original sequences $\left\{f_{n}\right\}$ and $\left\{g_{n}\right\}$ must converge locally uniformly to $f$ and $g$, respectively.

Now by Lemma $5.1,\left\{p_{n}\right\}$ and $\left\{p_{n}^{*}\right\}$ converge uniformly to the identity on $\partial \mathbb{D}$. Thus $\left\{g_{n} \circ p_{n}^{*}\right\}$ converges uniformly to $g$ on $\partial \mathbb{D}$.

Moreover,

$$
\left|l_{n}^{-1} u_{n}(z)-z\right| \leq \frac{M}{n}
$$

for all $z \in \partial \Omega_{n}$, by the Proximity Lemma. Hence we see

$$
l_{n}^{-1} u_{n} g_{n} p_{n}^{*} \rightarrow g
$$

uniformly on $\partial \mathbb{D}$ as well.

A similar argument implies

$$
f_{n} p_{n} \rightarrow f
$$

uniformly on $\partial \mathbb{D}$. However, it is easy to show that if $\eta_{n} \rightarrow \eta$ and $\xi_{n} \rightarrow \xi$ uniformly, and if $\xi^{-1}$ is uniformly continuous, then $\xi_{n}^{-1} \eta_{n}$ converges uniformly to $\xi^{-1} \eta$, where this composition is defined. As a result, we see

$$
\begin{aligned}
\varphi_{n} & =p_{n}{ }^{-1} \circ f_{n}^{-1} \circ l_{n}^{-1} \circ u_{n} \circ g_{n} \circ p_{n}^{*} \\
& =\left(f_{n} p_{n}\right)^{-1} \circ\left(l_{n}^{-1} u_{n} g_{n} p_{n}^{*}\right)
\end{aligned}
$$

converges to $f^{-1} g=\varphi$ uniformly on $\partial \mathbb{D}$.

Finally we observe that $\varphi$ is quasisymmetric on $\partial \mathbb{D}$ by Theorem 1.4. Since $\varphi$ is normalized as in Theorem 1.4, it must be the unique quasisymmetry corresponding to $\Gamma$.

\section{Proof of the Proximity and Extension Lemmas}

In general, if a packing is intersected with a Jordan domain, one would not expect the distance from the boundary of the carrier to Jordan curve to decrease with the mesh of the packing. For example, the curve could have infinitely many arbitrarily thin "necks," so that there would always be necks into which a packing could not reach. Of course, such a curve could not be a quasicircle. The geometry of our $K$-quasicircle $\Gamma$ and the combinatorics of our packings $P_{n}$ and $P_{n}^{*}$ will force the distance from $\partial \Omega_{n}$ and $\partial \Omega_{n}^{*}$ to $\Gamma$ to decrease to 0 as $n \rightarrow \infty$. In fact, we can even construct homeomorphisms which will move boundary points a distance which decreases to 0 as $n \rightarrow \infty$.

Proximity Lemma. Using the notation established above, for large $n$ there exist homeomorphisms $l_{n}: \partial \Omega_{n} \rightarrow \Gamma$ and $u_{n}: \partial \Omega_{n}^{*} \rightarrow \Gamma$ such that for all $z \in \partial \Omega_{n}$ and $w \in \partial \Omega_{n}^{*}$,

$$
\begin{array}{r}
\left|l_{n}(z)-z\right|<\frac{M}{2 n} \\
\left|u_{n}(w)-w\right|<\frac{M}{2 n},
\end{array}
$$


where $M$ depends only on $K$. In particular, we have

$$
\left|l_{n}^{-1} u_{n}(w)-w\right|<\frac{M}{n}
$$

Proof. First recall that by our construction in Section 3.1, each boundary vertex $v$ of $\Omega_{n}$ is connected to $\Gamma_{v} \in \Gamma$ by a line segment $\gamma_{v}$ of length at most $1 / n$. We may assume $\gamma_{v} \cap \Gamma=\left\{\Gamma_{v}\right\}$.

Next choose a boundary vertex $v_{1}$ of $\partial \Omega_{n}$. Let $A$ be the polygonal Jordan curve forming the boundary of $\Omega_{n}$. Parameterize $A$ over $[0,1]$ so that it is positively oriented with respect to $\Omega_{n}$ and $A(0)=v_{1}$. Similarly, parameterize $\Gamma$ over $[0,1]$ so that it is positively oriented with respect to $\Omega$ and $\Gamma(0)=\Gamma_{v_{1}}$.

Now we construct a finite sequence $v_{1}, v_{2}, \ldots, v_{p}$ of boundary vertices of $\Omega_{n}$ such that:

1. If $v_{i}=A\left(t_{i}\right), i=1,2, \ldots, p$, then $0=t_{1}<t_{2}<\cdots<t_{p}<1$.

2. If $\Gamma_{v_{i}}=\Gamma\left(s_{i}\right), i=1,2, \ldots, p$, then $0=s_{1}<s_{2}<\cdots<s_{p}<1$.

3. The segment $A\left(\left(t_{i}, t_{i+1}\right)\right), i=1,2, \ldots, p$, contains at most 36 boundary vertices of $\Omega_{n}$.

We adopt the convention that $v_{p+1}=v_{1}, t_{p+1}=t_{1}$, and $s_{p+1}=s_{1}$.

To construct our sequence, we start at vertex $v_{1}$ and proceed around $A$ in the positive direction until reaching a vertex $v_{2}$ such that $\gamma_{v_{1}} \cap \gamma_{v_{2}}=\emptyset$. Note that if a boundary vertex $w$ lies in $\mathrm{G}_{\mathcal{K}_{n}}\left(v_{1} ; 3\right)$, that is, if $w$ is 3 generation away from $v_{1}$, then faces containing $w$ do not intersect faces containing $v_{1}$. In particular, we have $\gamma_{v} \cap \gamma_{w}=\emptyset$. Since, for large $n, \mathrm{G}_{\mathcal{K}_{n}}\left(v_{1} ; 3\right) \neq \emptyset$, we can indeed find such a vertex which we label as $v_{2}$.

Note $\mathrm{G}_{\mathcal{K}_{n}}\left(v_{1} ; 1\right)$ and $\mathrm{G}_{\mathcal{K}_{n}}\left(v_{1} ; 2\right)$ can contain at most 6 and 12 vertices, respectively, since $\mathcal{K}_{n}$ was formed by deleting vertices from a constant degree 6 triangulation. The Jordan curve $A$ cannot hit a vertex twice; hence $A$ can hit at most 18 vertices before reaching $\mathrm{G}_{\mathcal{K}_{n}}\left(v_{1} ; 3\right)$. Consequently at most 18 vertices can lie on $A$ between $v_{1}$ and $v_{2}$.

Note that if $v_{2}=A\left(t_{2}\right)$ and $\Gamma_{v_{2}}=\Gamma\left(s_{2}\right)$, then $0=t_{1}<t_{2}<1$ and $0=s_{1}<s_{2}<1$.

Now suppose boundary vertices $v_{1}, v_{2}, \ldots, v_{m}$ of $\Omega_{n}$ have been chosen to satisfy conditions 1-3 above. Travel along $A$ positively from $v_{m}$ until encountering either

(A) the vertex $v_{1}$ or

(B) a vertex $v_{m+1}=A\left(t_{m+1}\right)$ such that $\gamma_{v_{m+1}} \cap \gamma_{v_{m}}=\emptyset$ and $\gamma_{v_{m+1}} \cap \gamma_{v_{1}}=\emptyset$.

Note that as described above, there can be at most 18 vertices $w$ such that $\gamma_{w} \cap \gamma_{v_{m}} \neq \emptyset$ and there can be at most 18 vertices $u$ such that $\gamma_{u} \cap \gamma_{v_{1}} \neq \emptyset$. Thus $A$ can hit at most 36 vertices before hitting one which satisfies our criterion (B). Hence, if $A$ first encounters $v_{1}$, then $A\left(\left(t_{m}, 1\right)\right)$ can contain at most 36 vertices and our construction is concluded.

Likewise, if $A$ first encounters a vertex $v_{m+1}=A\left(t_{m+1}\right)$ as described in condition (B), then $0=t_{1}<t_{2}<\ldots<t_{m}<t_{m+1}<1$, and we can be assured that $A\left(\left(t_{m}, t_{m+1}\right)\right)$ contains at most 36 boundary vertices.

Now $\Gamma_{v_{m+1}}=\Gamma\left(s_{m+1}\right)$, for some parameter value $s_{m+1}$. Observe that $\Gamma_{s_{m+1}}$ cannot equal $\Gamma\left(s_{1}\right)$ or $\Gamma\left(s_{m}\right)$ since $\gamma_{v_{m+1}} \cap \gamma_{v_{m}}=\emptyset$ and $\gamma_{v_{m+1}} \cap \gamma_{v_{1}}=\emptyset$. Hence $s_{m+1}$ cannot equal $0, s_{1}, s_{m}$, or 1 . 


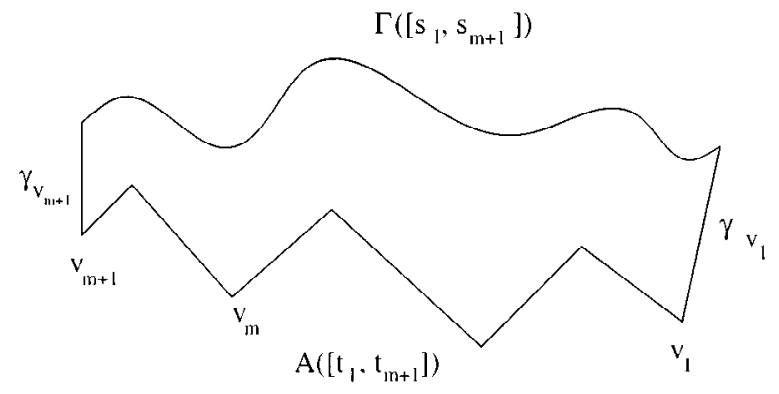

Fig. 6. The Jordan curve $J$.

Suppose that $0=s_{1}<s_{m+1}<s_{m}<1$. Let

$$
J=A\left(\left[t_{1}, t_{m+1}\right]\right) \cup \gamma_{v_{m+1}} \cup \Gamma\left(\left[s_{1}, s_{m+1}\right]\right) \cup \gamma_{v_{1}} .
$$

See Fig. 6.

Notice that by our construction

$$
\begin{aligned}
A \cap \gamma_{v_{1}} & =v_{1}=A\left(t_{1}\right), \\
A \cap \gamma_{v_{m+1}} & =v_{m+1}=A\left(t_{m+1}\right), \\
\Gamma \cap \gamma_{v_{1}} & =\Gamma_{v_{1}}=\Gamma\left(s_{1}\right), \\
\Gamma \cap \gamma_{v_{m+1}} & =\Gamma_{v_{m+1}}=\Gamma\left(s_{m+1}\right), \\
A \cap \Gamma & =\emptyset, \\
\gamma_{v_{1}} \cap \gamma_{v_{m+1}} & =\emptyset .
\end{aligned}
$$

Thus it is easy to see $J$ is a Jordan curve, and hence, by the Jordan Curve Theorem, $J$ divides the plane into two complementary components, one of which is bounded (the inside of $J$ ) and one of which is unbounded (the outside of $J$ ). Note that $J$ was constructed so as not to enclose any point of $\Omega_{n}$; that is, $\Omega_{n}$ must lie completely outside $J$.

Since $\gamma_{v_{m}}$ misses $\Gamma\left(\left[s_{1}, s_{m+1}\right]\right), \gamma_{v_{1}}$, and $\gamma_{v_{m+1}}$, we see

$$
\gamma_{v_{m}} \cap J=\gamma_{v_{m}} \cap A\left(\left[t_{1}, t_{m+1}\right]\right)=\left\{v_{m}\right\}
$$

Thus $\gamma_{v_{m}} \backslash\left\{v_{m}\right\}$ must lie entirely in one of the complementary components of $J$. However, near $v_{m}$ the curve $J$ forms a portion of $\partial \Omega_{n}$. Since $\gamma_{v_{m}} \cap \Omega_{n}=\left\{v_{m}\right\}$, then $\gamma_{v_{m}} \backslash\left\{v_{m}\right\}$ must lie inside $J$.

Similarly, $\Gamma\left(\left(s_{m+1}, 1\right)\right)$ does not intersect $J$ and must therefore lie entirely in one of the complementary components of $J$. However, our assumption that $s_{1}<s_{m+1}<s_{m}<1$ implies that $\Gamma\left(s_{m}\right) \in \Gamma\left(\left(s_{m+1}, 1\right)\right) \cap \gamma_{v_{m}}$. Hence $\Gamma\left(\left(s_{m+1}, 1\right)\right)$ must also lie inside $J$.

Nevertheless $\Gamma=\Gamma\left(\left[s_{1}, s_{m+1}\right]\right) \cup \Gamma\left(\left(s_{m+1}, 1\right)\right)$; consequently, every point of $\Gamma$ lies either on $J$ or inside $J$. Hence the bounded complementary component of $\Gamma$ must lie inside $J$. However, $\Omega_{n}$ both lies inside $\Gamma$ and outside $J$. This contradiction implies we must have $0=s_{1}<s_{m}<s_{m+1}<1$.

Thus by induction, we can construct a sequence $v_{1}, v_{2}, \ldots, v_{p}$ satisfying conditions $1-3$. 
Now since $\partial \Omega$ is a polygon, any point of $\partial \Omega$ can be written as $A\left((1-r) t_{i}+r t_{i+1}\right)$ for some $0 \leq r<1$ and some $i$. We define our map $l_{n}$ on $\partial \Omega_{n}$ by

$$
l_{n}\left(A\left((1-r) t_{i}+r t_{i+1}\right)\right)=\Gamma\left((1-r) s_{i}+r s_{i+1}\right) ;
$$

that is, we send each $v_{i}$ to $\Gamma_{v_{i}}$ and extend affinely in between. Since the sequence $\Gamma\left(s_{i}\right)$ proceeds positively around $\Gamma, l_{n}$ must be a homeomorphism.

Since there are at most 36 boundary vertices on $A\left(\left(t_{i}, t_{i+1}\right)\right)$ and adjacent vertices are separated by a distance of $1 / n$, we have

$$
\left|v_{i}-v_{i+1}\right|<\frac{37}{n}, \quad i=1,2, \ldots, p .
$$

Furthermore, if $z \in A\left(\left(t_{i}, t_{i+1}\right)\right)$, then

$$
\left|z-v_{i}\right|<\frac{1}{2} \frac{37}{n}
$$

Now the geometry of $\Gamma$ as a $K$-quasicircle comes into play. Between $l_{n}\left(v_{i}\right)$ and $l_{n}\left(v_{i+1}\right), \Gamma$ cannot wander too far away. That is, by Theorem 1.2 , there exists $R>1$ depending only on $K$, so that

$$
\operatorname{diam}\left(\Gamma_{l_{n}\left(v_{i}\right), l_{n}\left(v_{i+1}\right)}\right) \leq R\left|l_{n}\left(v_{i}\right)-l_{n}\left(v_{i+1}\right)\right|,
$$

where $\Gamma_{l_{n}\left(v_{i}\right), l_{n}\left(v_{i+1}\right)}$ is the subarc of $\Gamma$ connecting $l_{n}\left(v_{i}\right)$ and $l_{n}\left(v_{i+1}\right)$ having the smaller diameter.

Consequently,

$$
\begin{aligned}
\left|l_{n}(z)-z\right| & \leq\left|l_{n}(z)-l_{n}\left(v_{i}\right)\right|+\left|l_{n}\left(v_{i}\right)-v_{i}\right|+\left|v_{i}-z\right| \\
& <\operatorname{diam}\left(\Gamma_{l_{n}\left(v_{i}\right), l_{n}\left(v_{i+1}\right)}\right)+\frac{1}{n}+\frac{37}{2 n} \\
& \leq R\left|\Gamma_{l_{n}\left(v_{i}\right), l_{n}\left(v_{i+1}\right)}\right|+\frac{39}{2 n} \\
& \leq R\left(\left|\Gamma_{v_{i}}-v_{i}\right|+\left|v_{i}-v_{i+1}\right|+\left|v_{i+1}-\Gamma_{v_{i+1}}\right|\right)+\frac{39}{2 n} \\
& <R\left(\frac{1}{n}+\frac{36}{n}+\frac{1}{n}\right)+\frac{39}{2 n} R \\
& \leq \frac{117 R}{2 n} .
\end{aligned}
$$

The maps $u_{n}$ can be constructed in precisely the same manner. Conditions (1) and (2) now follow with $M=117 R$.

The maps $l_{n}$ and $u_{n}$ now allow us to compare the geometry of $\partial \Omega_{n}$ and $\partial \Omega_{n}^{*}$ to that of $\Gamma$. In particular, we see $\partial \Omega_{n}$ and $\partial \Omega_{n}^{*}$ are also quasicircles.

Extension Lemma. For each $n, \partial \Omega_{n}$ and $\partial \Omega_{n}^{*}$ are $K^{\prime}$-quasicircles, where $K^{\prime}$ depends only on $K$. In particular, there exist $\left(K^{\prime}\right)^{2}$-quasiconformal reflections $J_{n}$ and $J_{n}^{*}$ in $\partial \Omega_{n}$ and $\partial \Omega_{n}^{*}$, respectively. 
Proof. We give the proof for $\partial \Omega_{n}$; the proof for $\partial \Omega_{n}^{*}$ is of course the same.

As in the proof of the Proximity Lemma, we let $A$ denote the Jordan curve forming $\partial \Omega_{n}$. Fix $n$ and $w, z \in A$. As in the statement of Theorem 1.2, we let $A_{w, z}$ denote the subarc of $A$ connecting $w$ and $z$ and having the smaller diameter.

Note that by the Proximity Lemma, for all $a, b \in A_{w, z}$,

$$
\begin{aligned}
|a-b| & \leq\left|a-l_{n}(a)\right|+\left|l_{n}(a)-l_{n}(b)\right|+\left|l_{n}(b)-b\right| \\
& <\frac{M}{2 n}+\operatorname{diam}\left(l_{n}\left(A_{w, z}\right)\right)+\frac{M}{2 n} \\
& =\frac{M}{n}+\operatorname{diam}\left(l_{n}\left(A_{w, z}\right)\right) .
\end{aligned}
$$

Thus

$$
\operatorname{diam}\left(A_{w, z}\right)<\frac{M}{n}+\operatorname{diam}\left(l_{n}\left(A_{w, z}\right)\right) .
$$

Unfortunately, even though $A_{w, z}$ is the shorter subarc connecting $w$ and $z$, it is not necessarily the case that $l_{n}\left(A_{w, z}\right)$ is the shorter subarc connecting $l_{n}(w)$ and $l_{n}(z)$; that is, we need not have $\Gamma_{l_{n}(w), l_{n}(z)}=l_{n}\left(A_{w, z}\right)$.

However, if $\Gamma_{l_{n}(w), l_{n}(z)} \neq l_{n}\left(A_{w, z}\right)$, then $\Gamma_{l_{n}(w), l_{n}(z)}=l_{n}\left(A_{w, z}^{c}\right)$, where $A_{w, z}^{c}$ is the subarc of $A$ connecting $w$ and $z$ and having the larger diameter. In this case, we see that for all $a, b \in A_{w, z}^{c}$,

$$
\begin{aligned}
|a-b| & \leq\left|a-l_{n}(a)\right|+\left|l_{n}(a)-l_{n}(b)\right|+\left|l_{n}(b)-b\right| \\
& <\frac{M}{n}+\operatorname{diam}\left(l_{n}\left(A_{w, z}^{c}\right)\right) \\
& =\frac{M}{n}+\operatorname{diam}\left(\Gamma_{l_{n}(w), l_{n}(z)}\right) .
\end{aligned}
$$

Thus

$$
\begin{aligned}
\operatorname{diam}\left(A_{w, z}\right) & \leq \operatorname{diam}\left(A_{w, z}\right) \\
& <\frac{M}{n}+\operatorname{diam}\left(\Gamma_{l_{n}(w), l_{n}(z)}\right) .
\end{aligned}
$$

Of course, if $\Gamma_{l_{n}(w), l_{n}(z)}=l_{n}\left(A_{w, z}\right)$, then we still have $\operatorname{diam}\left(A_{w, z}\right)<M / n+$ $\operatorname{diam}\left(\Gamma_{l_{n}(w), l_{n}(z)}\right)$ by (6). Thus in either case,

$$
\begin{aligned}
\operatorname{diam}\left(A_{w, z}\right) & <\frac{M}{n}+\operatorname{diam}\left(\Gamma_{l_{n}(w), l_{n}(z)}\right) \\
& <\frac{M}{n}+R\left|l_{n}(z)-l_{n}(w)\right| \\
& \leq \frac{M}{n}+R\left(\left|l_{n}(z)-z\right|+|z-w|+\left|w-l_{n}(w)\right|\right) \\
& \leq \frac{M}{n}+R\left(\frac{M}{2 n}+|z-w|+\frac{M}{2 n}\right) \\
& \leq \frac{M}{n}+\frac{M R}{n}+R|z-w| \\
& \leq \frac{R(M+1)}{n}+R|z-w| .
\end{aligned}
$$




$$
\text { If }|z-w| \geq 1 / n \text {, then (7) becomes }
$$

$$
\begin{aligned}
\operatorname{diam}\left(A_{w, z}\right) & <\frac{R(M+1)}{n}+R|z-w| \\
& \leq R(M+1)|z-w|+R|z-w| \\
& =R(M+2)|z-w| .
\end{aligned}
$$

Recall that $\partial \Omega_{n}$ was formed from the union of sides of equilateral triangles of side length $1 / n$. Thus, if $|z-w|<1 / n$, then $z$ and $w$ must lie either on a euclidean line segment of length $1 / n$ or on two different line segments of length $1 / n$ which meet at angle $\pi / 3$ or $2 \pi / 3$. In either case, clearly

$$
\operatorname{diam}\left(A_{w, z}\right) \leq 2|z-w|<R(M+2)|z-w|
$$

Thus by Theorem 1.2, $A=\partial \Omega_{n}$ is a $K^{\prime}$-quasicircle, where $K^{\prime}$ depends only on $M$ and $R$ (and thus only on $K$ ). The second assertion of the lemma then follows from Theorem 1.2 as well.

\section{Acknowledgments}

This work was completed as part of the author's Ph.D. thesis under the direction of Professor Ken Stephenson. The author expresses his most sincere thanks to Professor Stephenson for his assistance and encouragement.

\section{References}

1. L. Ahlfors, Quasiconformal reflections, Acta Math. 109 (1963), 291-301.

2. L. Ahlfors, Lectures on Quasiconformal Mappings, van Nostrand, Princeton, NJ, 1966.

3. E. M. Andreev, Convex polyhedra of finite volume in Lobacevskii space, Math. USSR-Sb. 12 (1970), 255-259 (English).

4. A. F. Beardon and K. Stephenson, The uniformization theorem for circle packings, Indiana Univ. Math. J. 39 (1990), 1383-1425.

5. A. Beurling and L. Ahlfors, The boundary correspondence under quasiconformal mappings, Acta Math. 96 (1956), 125-141.

6. P. L. Bowers and K. Stephenson, The set of circle packing points in the Teichmüller space of a surface of finite conformal type is dense, Math. Proc. Cambridge Philos. Soc. 111 (1992), 487-513.

7. C. Collins and K. Stephenson, A circle packing algorithm, preprint.

8. T. Dubejko, Approximation of analytic functions with prescribed boundary conditions by circle packing maps, Discrete Comput. Geom. 17 (1997), 67-77.

9. T. Dubejko and K. Stephenson, Circle packing: experiments in discrete analytic function theory, Experiment. Math. 4(4) (1995), 307-348.

10. R. J. Duffin, Basic properties of discrete analytic functions, Duke Math. J. 23 (1956), 335-363.

11. R. J. Duffin, Potential theory on a rhombic lattice, J. Combin. Theory 5 (1968), 258-272.

12. W. J. Floyd, J. W. Cannon and W. R. Parray, Squaring Rectangles: the Finite Riemann Mapping Theorem, Contemporary Mathematics, vol. 169, American Mathematical Society, Providence, RI, 1994, pp. 133212.

13. F. P. Gardiner and N. Lakic, Quasiconformal Teichmüller Theory, Mathematical Surveys and Monographs, vol. 76, American Mathematical Society, Providence, RI, 2000. 
14. F. W. Gehring, Characteristic properties of quasidisks, Les Presses De L'Universiteé De Montréal, Montréal, 1982.

15. Z.-X. He, An estimate for hexagonal circle packings, J. Differential Geom. 33 (1991), 395-412.

16. Z.-X. He, Rigidity of infinite disk patterns, Ann. of Math. 2 (1999), 1-33.

17. Z.-X. He and B. Rodin, Convergence of circle packings of finite valence to Riemann mappings, Comm. Anal. Geom. 1 (1993), 31-41.

18. A.-X. He and O. Schramm, On the convergence of circle packings to the Riemann map, Invent. Math. 125 (1996), 285-305.

19. L. H. Howell and L. N. Trefethen, A modified Schwarz-Christoffel transformation for elongated regions, SIAM J. Sci. Statist. Comput. 11(5) (1990), 928-949.

20. J. A. Kelingos, Boundary correspondence under quasiconformal mappings, Michigan Math. J. 13 (1966), 235-249.

21. P. Koebe, Kontaktprobleme der Konformen Abbildung, Ber. Sächs. Akad. Wiss. Leipzig, Math.-Phys. Kl. 88 (1936), 141-164.

22. J. G. Krzyż, Quasicircles and harmonic measure, Ann. Acad. Sci. Fenn. Ser. A I Math. 12 (1987), 19-24.

23. J. G. Krzyż, On the notion of the universal Teichmüller space, Rev. Roumaine Math. Pures Appl. 40(2) (1995), 169-175.

24. O. Lehto, Univalent Functions and Teichmüller Spaces, Springer-Verlag, Berlin, 1987.

25. O. Lehto and K. I. Virtanen, Quasiconformal Mappings in the Plane, second edition, Springer-Verlag, Berlin, 1973.

26. S. Nag, Matrices: canonical mappings in string theory, Rev. Roumaine Math. Pures Appl. 39(8) (1991), 789-827.

27. O. Pekonen, The interface of Teichmüller theory and string theory, Rev. Roumaine Math. Pures Appl. 38(8) (1994), 829-854.

28. O. Pekonen, Universal Teichmüller space in geometry and physics, J. Geom. Phys. 15 (1995), $227-251$.

29. B. Rodin and D. Sullivan, The convergence of circle packings to the Riemann mapping, J. Differential Geom. 26 (1987), 349-360.

30. O. Schramm, Rigidity of infinite (circle) packings, J. Amer. Math. Soc. 4 (1991), 127-149.

31. O. Schramm, Circle patterns with the combinatorics of the square grid, Duke Math. J. 86 (1997), 347-389.

32. K. Stephenson, Circle packing and discrete analytic function theory, preprint.

33. K. Stephenson, A probabilistic proof of Thurston's conjecture on circle packings, Rend. Sem. Mat. Fis. Milano 66 (1996), 201-291.

34. K. Stephenson, The approximation of conformal structures via circle packing, in Computational Methods and Function Theory (N. Papamichael, S. Ruscheweyh, and E.B. Saff, eds.), Series in Approximations and Decompositions, World Scientific, Singapore, 1997, pp. 551-582.

35. W. Thurston, The geometry and topology of 3-manifolds, Princeton University Notes, preprint.

36. W. Thurston, The finite Riemann mapping theorem, 1985, Invited talk, An International Symposium at Purdue University on the Occasion of the Proof of the Bieberbach Conjecture, March 1985.

37. M. Tienari, Fortsetzung einer quasikonformen Abbildung über einen Jordanbogen, Ann. Acad. Sci. Fenn. A I Math. 321 (1962), 32pp.

38. G. B. Williams, Discrete conformal welding, preprint.

39. C. Zemach, A conformal map formula for difficult cases, J. Comput. Appl. Math. 14 (1986), 207-215.

Received May 19, 1999, and in revised form April 6, 2000. Online publication September 6, 2000. 